\title{
ИССЛЕДОВАНИЕ АНИОНООБМЕННЫХ СМОЛ ДЛЯ ПРИМЕНЕНИЯ В ГЕНЕРАТОРНОЙ СИСТЕМЕ 44TI/44SC
}

\section{Н.А. Титченко, Б.В. Егорова, А.О. Якушева, С.Н. Калмыков}

Химический факультет, Московский государственный университет им. М.В. Ломоносова, 119991, Российская Федерация, г. Москва, Ленинские горы, д. 1.

DOI: 10.19163/MedChemRussia2021-2021-279

E-mail: nikolaytitchenko@yandex.ru

Скандий является перспективным элементом для применения в ядерной медицине, он обладает тераностической парой: диагностическим ${ }^{44} \mathrm{Sc}$ $\left(\beta+, T_{1 / 2}=3,97\right.$ ч) и терапевтическим ${ }^{47} \mathrm{Sc}\left(\beta-\mathrm{T}_{1 / 2}=3,35\right.$ дня). Одним из способов получения ${ }^{44} \mathrm{Sc}$ является использование генераторной системы ${ }^{44} \mathrm{Ti} /{ }^{44} \mathrm{Sc}$. Преимуществом этого метода перед получением на циклотроне является высокая радионуклидная чистота за счёт отсутствия примеси ${ }^{44 \mathrm{~m}} \mathrm{Sc}\left(\mathrm{T}_{1 / 2}=2,44\right.$ дня). В работах [1,2] предложен генератор на основе анионообменной смолы AG $1 \times 8$ с последующей обработкой элюата.

В данной работе были исследованы генераторные системы на основе анионообменных смол DOWEX 1-X8 и TEVA (TrisKem Int.). В качестве элюента был использован раствор 0,005 $\mathrm{M} \mathrm{H}_{2} \mathrm{C}_{2} \mathrm{O}_{4}+0,065 \mathrm{M} \mathrm{HCl}$, предложенный в литературе для AG 1x8 [1]. Для перевода полученного скандия в пригодную для радиофармацевтического синтеза среду использовали систему, предложенную в [2]: катионообменная смола DOWEX 50-Х8, элюент 0,5 M NH $\mathrm{pH}=4,0$. Дополнительно данный процесс был исследован при скоростях элюирования 0,7 мл/мин и 0,2 мл/мин, а также при $\mathrm{pH}=3,5 / 4 / 5$. Возможность использования ${ }^{44} \mathrm{Sc}$, полученного данным способом, была подтверждена путём синтеза комплексов ${ }^{44} \mathrm{Sc} \mathrm{c} \mathrm{DOTA-конъюгатами} \mathrm{коротких} \mathrm{пептидов.}$

Было показано, что элюент 0,005 $\mathrm{M} \mathrm{H}_{2} \mathrm{C}_{2} \mathrm{O}_{4}+0,065 \mathrm{M} \mathrm{HCl}$ является пригодным для создания генератора на основе TEVA. Генераторная система на основе TEVA является более эффективной, чем DOWEX 1х8 и позволяет получить ${ }^{44} \mathrm{Sc} \mathrm{c} \mathrm{выходом} \mathrm{98,8 \% ,} \mathrm{объём} \mathrm{элюата} 5$ мл. Обработку элюента предпочтительнее проводить при рН = 4 и скорости элюирования 0,2 мл/мин. Выход составил 38\%, объём элюата 0,5 мл. Полученный скандий может быть применён в радиофармацевтическом синтезе.

\section{Литература}

[1] Filosofov, D. V., N. S. Loktionova, and Frank Rösch. «A 44Ti/44Sc radionuclide generator for potential application of 44Sc-based PET-radiopharmaceuticals.» rca-Radiochimica Acta 98.3 (2010): 149-156.

[2] Pruszyński, M., et al. «Post-elution processing of 44Ti/44Sc generator-derived 44Sc for clinical application.» Applied Radiation and Isotopes 68.9 (2010): 1636-1641. 